\title{
The needs of family members of intensive care unit patients: A grounded theory study
}

\author{
J de Beer, ${ }^{1} \mathrm{PhD} ; \mathrm{P}$ Brysiewicz, ${ }^{2} \mathrm{PhD}$ \\ ${ }^{1}$ Critical Care Nursing, School of Nursing, University of KwaZulu-Natal, Durban, South Africa and College of Nursing- \\ Jeddah, King Saud bin Abdul Aziz University for Health Sciences, Saudi Arabia \\ ${ }^{2}$ Emergency and Critical Care Nursing, School of Nursing, University of KwaZulu-Natal, Durban, South Africa
}

\section{Corresponding author: J de Beer (beerje@ngha.med.sa)}

\begin{abstract}
Background. The unexpected admission of a loved one to an intensive care unit (ICU) may have a negative effect on the everyday lives of family members, as they have had little time to adjust. Hence, it is imperative for healthcare professionals to promote optimal outcomes for both the patient and family members during admission for critical illness.

Objective. To explore and describe the needs of families during critical illness and to develop methods to provide family care during a critical illness of a loved one.

Methods. The Strauss and Corbin grounded theory approach was used. In-depth interviews with 16 intensive care nurses, 6 doctors and 9 family members in private and public settings were completed.

Results. Five codes emerged using the characteristic coding in grounded theory. These were identified as information sharing; reassurance; striving for consolation; garnering of resources; and cultural and religious co-operation.

Conclusion. This study elicited the needs of family members of ICU patients. Methods tailored around these needs were presented to support family members during a critical illness.
\end{abstract}

S Afr J Crit Care 2016;32(2):44-49. DOI:10.7196/SAJCC.2016.v32i2.298

The sudden admission of a loved one to an intensive care unit (ICU) leads to chaos, worry and stress for both the patient and family members (FMs). This results in a limited ability to adapt to the situation, as FMs are faced with a traumatic, unexpected and difficult situation, which is further compounded by shock and uncertainty of not knowing whether the outcome for their loved one will be survival, disability or death. ${ }^{[1]}$ According to Pryzby, ${ }^{[2]}$ 'families are in a sense dysrhythmic' and may display erratic thinking following the admission of a loved one to an ICU. Other authors suggest that this emergent situation (unpredicted and severe illness followed by hospitalisation) throws FMs into disequilibrium and renders them uncertain as to how to respond. Disruptions in the roles of FMs may occur, forcing them to reorganise and change roles to regain equilibrium. ${ }^{[3,4]}$

Attending to a critically unstable patient being admitted to intensive care takes precedence over addressing the psychological turmoil experienced by their FMs. However, addressing the psychological distress of FMs remains an integral part of the comprehensive intensive care approach. FMs often play a significant role in promoting the psychological wellbeing of a critically ill patient by their familiar and caring presence, their meaningful interaction with the patient, and their collaboration with the healthcare team in caring for the patient. However, a family's ability to support the patient may be compromised by their own psychological distress. ${ }^{[5]}$

Although significant progress has been made to incorporate nursing care from a patientcentred approach to one with an increased family-centred focus, it is more difficult in an ICU because of the intensity of the environment, where factors such as technology and complexity of care are prioritised over relational approaches. ${ }^{[6,7]}$ It is even more challenging in a multicultural context, as dealing with multicultural FMs in an intensive care environment presents particular difficulties for healthcare professionals. ${ }^{[8]}$

Although many models of providing family care have been developed internationally, there seems to be a gap in the available literature regarding family support in South African (SA) ICUs. Extensive research has been conducted internationally, which has produced results showing that integrating FMs into ICUs is important, not only for providing holistic care to patients, but also for contributing to a decrease in the stress levels of both patients and FMs. ${ }^{[2]}$ There are currently no documented family care programmes/policies or strategies available in SA, and against this backdrop we decided to explore the phenomenon of family care during critical illness and to identify possible support methods. This was a large study, with the purpose of developing a strategy for the enhancement of such care in ICUs in Durban, KwaZulu-Natal, SA. This article, however, reports on the objectives and focuses on the methods used to provide family care.

\section{Methods}

The research method used was a grounded theory approach as described by Strauss and Corbin. ${ }^{[9]}$ Grounded theory was employed as the methodological approach best suited to elucidate connections between concepts that emerged from the data, thus enabling the development of an inductively derived explanatory theory about a phenomenon. ${ }^{[10]}$ The Strauss and Corbin version was chosen, as this method provided more structure for the primary researcher, who was a novice in qualitative research.

The study was conducted at two hospitals in Durban. Hospital A is a 807-bed tertiary referral public hospital with 10 ICUs. Hospital $\mathrm{B}$ is a 400-bed private hospital with three ICUs. These hospitals were selected for their accessibility to the researchers. 
Participants included intensive care nurses (ICNs), doctors and FMs present in the ICU during the study. Within the context of this study, FMs included a spouse, parent, or child ( $\geq 18$ years of age) or any person(s) who played a significant role in the patient's life, including a person(s) not legally related to the patient.

Sampling began with purposive voluntary sampling, which included choosing participants who were available in the ICUs at the time of data collection. This was followed by theoretical sampling until theoretical saturation was reached and no new data emerged from analysis. The sample size consisted of 16 ICNs, 6 doctors and 9 family members, giving a total of 31 participants from the 13 ICUs in the two hospitals (17 from the public setting and 14 from the private setting).

Data collection using in-depth interviews commenced after permission was obtained from the relevant authorities of the hospitals and the Ethics Committee of the University of KwaZulu-Natal (ref. no.: HSS/0102/10). Interviews were conducted in English in a private area of the ICU and lasted between 30 and 45 minutes. Participants were provided with an information document in either English or isiZulu. They were made aware that participation was voluntary and that they had the right to withdraw at any time. Written permission was obtained from participants, and their permission was also obtained to tape record the interviews. Owing to the sensitive nature of the data collected, the researcher had ensured that a social worker or psychologist would be available if any of the FMs required counselling or debriefing.

We followed the strategies of credibility, dependability, confirmability and transferability to ensure trustworthiness, ${ }^{[11]}$ and used peer debriefing for external validation of findings. Member checking was done to ensure credibility, which involved providing feedback about the emerging data and interpretation to participants to ensure that it was a true reflection of their reactions. The study participants did not suggest any changes. For dependability, the researchers provided details of the methodology to allow for the replication of the study. A confirmability trail was also established by the researcher recording the research activities over time so that others could follow the research process undertaken.

\section{Analysis}

The analysis of data began by using the process of coding as soon as the first interview had been completed. Coding is described as the first analytical step, which generates the skeleton of analysis, and is characterised by three phases: open, axial and selective coding, with constant comparison taking place during each phase. Data were analysed manually. As data collection and data analysis are interwoven in grounded theory, it was found that most of these steps occurred simultaneously as the researcher moved back and forth between data collection and analysis. Inherent in the data analysis process was the process of theoretical sensitivity, which refers to the ability to recognise what is important in the data, helping to generate data that are faithful to the phenomenon being studied. This was particularly important in the context of this study as, being an ICN, it was vital that the researcher was aware of the importance of the subjective self. This was achieved by creative confrontation, i.e. co-constructing meaning with the participants of this study (the constructivist paradigm, which was in line with the researcher's paradigm). Data analysis began with open coding after the first interview had been transcribed. During open coding the researcher repeatedly read the transcripts to become familiar with the text to enhance the coding and analysis process. This involved looking at each line and allocating concepts to words or phrases. The data were also broken down into incidents and compared with other incidents and other data to develop the concepts. The concepts were then analysed and those relating to a common theme were assigned together to find higher commonalities, i.e. categories, thereby reducing the number of concepts.

Open coding followed axial coding, which involved examining the full list of categories produced at the end of open coding. Labelled categories were grouped together under the same headings. These categories were further analysed by assessing them individually in terms of their dimensions and properties. This allowed sub-categories to emerge on the basis of how they related to the category in question.

Selective coding followed axial coding, which involved the process of selecting the core category, systematically relating it to other categories, validating those relationships, and filling in categories that needed further refinement and development.

During data analysis, the iterative process of analysis was also used, which allowed the researcher to repeatedly study the data and ask questions. The researcher also used the constant comparative methods for data analysis, especially the technique of 'waving a red flag', which helped her discern beyond what was obvious in the data. ${ }^{[9]}$

\section{Results}

The findings are presented in codes as generated by the data. The following codes discuss the methods to provide family care during critical illness based on the needs of FMs as highlighted by participants.

\section{Code 1: Information sharing}

Most participants emphasised that the most essential need was the need to know, as it was an important part of making what happened intelligible and manageable. Information shared with FMs should be at a level that they can understand:

ICN: 'Some populations are not educated, are not well read ... their level of understanding, their knowledge level is lower, ... it's a literacy level ... you would need to communicate with them in a manner that they understand.' FM (husband): 'I needed information .... to be able to understand what's happening to know what's happening could have helped me to manage the situation better.'

The findings also suggest that FMs needed to know what is being done for the patient, what the medical treatment entailed, why the patient needed to undergo specific procedures, details of the patient's progress and technical support that the patient received:

FM (wife): 'I would like to know what is his blood pressure, where on the machine is his pulse, everything, so that they tell you then you can look for yourself. There is nothing worse that when the machine is buzzing and you don't know what to do .... ?

Medical doctor: 'They just need somebody to explain things to them ... they come in and see their loved ones with a tube in their mouth or nose, not responding and a whole lot of drugs and even if the patient is getting better. It is the first thing that they are seeing, the patient, they think the worst.'

Participants stressed the need for orientation in the technical context of an ICU, as the environment itself was very challenging. It emerged that the harsh environment of the machines, monitors and constantly beeping alarms instilled feelings of inadequacy in FMs:

FM (sister): 'We heard machines beeping, we were panicking, we thought maybe the person was dying so we had to call a nurse and ask her to check up on him because 
we were anxious and did not know what was going on ... it would be nice if someone could tell us about all the machines and monitors.'

ICN: 'I think being admitted to an ICU is frightening, whether you're stable or ... - not to us - it's an everyday thing, it's not something new, but for the layman out there it's something frightening. Just the word ICU, it is a scary environment ... there's monitors; you might have a person on a life support machine. There is a whole lot of vacolitres running - it's very frightening ... and it is important to orientate them to the environment.'

Participants verbalised that information sharing should also include the provision of literature, as it would provide FMs continuous access to information whenever they needed it:

FM (wife): 'I think literature would be a great help. People can read and understand and see the pictures as well and see that, ok this line means that and $j a$, I think that would be a great help to constantly have that information reinforced.' Medical doctor: '... that all the relatives have access to the information and don't have to go and find out things on their own, because they don't know the precise or exact sites to get this information ... an intensive care should have free access to the internet both for the nurse and doctors so that written information can be referred to at a later stage.'

Participants suggested that information such as visiting hours, hospital phone numbers, the nature of technical support (ventilators (lifesupport machines), endotracheal tubes, cardiac monitors, sedation), the hospital coffee shop, hospital chaplains and access to telephones should be available in writing:

FM (husband): 'It would be beneficial to access information on the visiting hours, telephone numbers of the hospital and what all the machines are for in the ICU, like the lifesupport machine, the heart machine, the breathing tube, where the public phones are and coffee shops and a prayer is available.'

Participants also reported that the sharing of honest, factual information was vital, even if the news was not always positive:

ICN: 'It is of the utmost importance to educate them ... in continuously educating them, explaining to them each procedure done. I will have to make sure that honest accurate information is shared, non-maleficence, beneficence all that is important, we need to respect that.' Medical doctor: 'He [FM] will tell you that they want to hear everything ... so you have to tell them everything and not hide anything, even if the patient is not for resuscitation, withdrawal of treatment or issues such as brain death.'

There was a need for clear, concise information devoid of inconsistencies. Many participants commented that they appreciated it when the team of healthcare professionals treating the patient provided a unified message rather than various individuals giving conflicting information:

FM (daughter): 'Sometimes the doctors conflict what they say, especially if there is more than one doctor seeing the patient. It would be nice if they could have a chat among themselves and tell us one story'
Participants stressed that information should be given patiently and sensitively, especially with regard to end-of-life decisions, poor prognosis and functional disabilities of the patient:

FM (mother): '... they have contacted me and told me that he is brain dead and the doctor just came and told us, you know he just told us sorry you know your child is brain dead, you know, with no compassion ....'

\section{Code 2: Reassurance}

The vigilant attendance of FMs demonstrated their need for reassurance and the need to be close to the patient to develop confidence and trust in the care being provided by healthcare professionals:

FM (sister): '... but we did not leave the hospital until we knew that the operation was successful. We also did not want to leave as we needed to see him and make sure that he was okay, that he was taken care of. We needed to see for ourselves.'

Participants explained that vigilant attendance was also a way of ensuring that the patient's dignity was maintained. FMs wanted to be present to ensure that their loved one was clean, covered at all times and free from pain:

ICN: 'He was jumping out the bed ... the wife who was there ... helped her husband, covering him up so that he does not expose himself to everyone.'

FMs felt that vigilant attendance was a means of providing psychological support for the patient. Even though the patients were often unable to communicate or were unconscious, participants felt that they had contributed to their emotional care by being close. They felt that this was extremely important for providing hope for survival to the patient:

Medical doctor: 'They also need to be given the opportunity to be closer to the patient, to be able to provide that support to the patient ... This sometimes creates a source of hope for the patient, even if the patient is sedated or unconscious.'

\section{Code 3: Striving for consolation}

In the context of this study, striving for consolation refers to family members' need for emotional support. It emerged that FMs needed each other as well as close friends or neighbours for support:

FM (husband): 'By having your family present, it gives you an opportunity to share your feelings and receive support instead of trying to manage this on your own. It is always helpful to receive the support of family and friends, sometimes getting all the FMs together is helpful, they provide an unconditional source of support.'

FM (wife): '... I have the family everyday phoning, listen, have you eaten breakfast, did you eat lunch, what time are you going to bed? Make sure that you get a good sleep. If you need anything, we are here for you. That type of thing. I tell you they have been very good.'

Participants reported receiving support from FMs of other patients. This was usually seen in cases where FMs did not receive support from their own FMs, as the hospital was far from home:

FM (daughter): 'Some of the patients' FMs were very good to me as well. I mean the one lady that was here with her daughter always came to see me, because they saw I was on my own.' 
Participants indicated that receiving consolation sometimes necessitated making use of outside support, such as the services of a counsellor, psychologist or religious leader:

FM (mother): ' ... being counselled helps ... you know ICUs should have a service provider like that you know; you can counsel FMs and maybe the patients themselves.'

\section{Code 4: Garnering of resources}

FMs were involved in garnering of non-material resources that included the need to maintain hope with regard to the patient's condition and progress. FMs found ways of detecting hints of reaction from the patient, such as a slight movement of the hand or eye, a cough or moving of the lips. This signalled hope that the patient was able to sense their presence and that there was a chance of recovery:

FM (daughter): 'The doctor says that ... is not doing well ... mum was coughing when the nurse was moving the tube in her mouth. Even if she moves a hand, eye or lips, this will give us hope as this means that she is aware of our presence.'

It also emerged that FMs tapped into physical resources provided by the hospital to meet their needs. Such needs included comfortable seats in the waiting area and a place to sleep in instances of FMs travelling long distances to the hospital. Participants mentioned that comfortable seating was a priority, as they might spend long hours in the waiting area:

ICN: 'We need to have comfortable seating arrangements in the waiting area, as FMs spend many hours there. Sometimes we try to accommodate FMs that are travelling far, especially if the patient is a paediatric, by providing a sleeping area.' ICN: 'We tell FMs about the coffee... coffee place downstairs, the canteen, so they can go have cups of coffee and all of that. Also depending on the situation we will make them coffee out of our own here.'

The findings of this study highlighted the importance of having a private space to accommodate FMs who are emotionally distraught. Where no such space is available, healthcare professionals have to talk to FMs in public areas, where displays of emotion and crying cause fear and anxiety to the FMs of other seriously ill patients:

ICN: 'We don't have a waiting room for FMs ... so if the relatives are crying and there are other relatives just standing around it becomes hectic and some other FMs might freak out when they hear about their loved ones. We end up talking to them in the open where other FMs may hear what you talking about ... it is very important to provide a private space.'

\section{Code 5: Cultural and religious co-operation}

It emerged that ICNs and doctors displayed a large degree of cultural sensitivity during the critical illness of patients. Cultural sensitivity in this instance referred to ICNs and doctors taking into account the cultural values, beliefs, lifestyles and practices of patients and their FMs. In the context of this study, it also referred to ICNs and doctors setting aside their own cultural values to understand the culture of the patient and FMs. ICNs and doctors reported the importance of acknowledging the diverse cultures of FMs, as different cultures had different ways of dealing with the stress of an ICU admission.

FM (wife): '... I prayed for him, turned ashes and salt to ward off the evil eye ... and the staff respected this ... it is very important to be respected culturally'
ICN: 'Well, you got to have a basic understanding of the different cultures, and what is acceptable and what is not acceptable in that cultural group ... Whether you agree with it or not, is not the point ..., but you've got to show respect.' Medical doctor: '... where we have to be sensitive regarding, we live in quite a diverse society, so we have to respect cultural, different cultural practices.'

It became apparent that some FMs relied on cultural beliefs and rituals to cope with the situation. ICNs and doctors reported that they tried to accommodate FMs' cultural rituals and beliefs by allowing them to pin charms, amulets and photographs of holy figures to the pillows of the patients or next to their heads, provided it did not harm them:

ICN: 'We also had, like the Indian community which has a lot of rituals and cultures and prayers that they would like to do. We also respect them in that situation and also they bring certain kinds of ... prayer amulets and like souvenirs, statues, charms and religious photographs ... which are meant to ward off evil spirits and protect ... as long as it does not harm the patient.'

In instances where the patient dies, Zulu FMs wish to return to the ICU with a tree branch to 'collect the spirit' of their dead FM. It seems this can be done days or weeks after the actual death of the patient:

ICN: 'In the African culture, where someone's died, we allow them to come in with their branches, we allow them to take their, hmm, spirit with them.'

Medical doctor: 'I would say with culture, it's coming to fetch the spirit, it's a new experience ... doing that is something we must allow people to do.'

ICNs and doctors reported that, in many cases, the bed or cubicle in which the patient had died was already occupied by a new patient. Having strangers enter the room with branches, created awkwardness and could be frightening for the new patient and their FMs. Despite this, however, all requests to collect spirits were usually accommodated. ICNs and doctors also reported that it was important to accommodate the needs of Muslim patients who, according to their customs, must be buried on the day they die:

Medical doctor: '... the same goes for arranging postmortems for the Islamic patients that need to be buried the same day.'

ICN: 'In the African culture, where someone has died, we allow them [FMs] to come in with their branches to fetch the spirit ... but it can be quite scary if the room is occupied by a another patient when the FMs are collecting the spirit.'

Muslims and Hindus considered it a cultural duty to visit a relative in ICU as a means of providing support to the patient and close family members. This means that large numbers of visitors may arrive during visiting hours to see the critically ill patient:

ICN: 'As you have noticed that most of our visitors are Indians, and we have FMs coming from all parts of the country ... We have one lounge here ... We have to understand that they want to show their support.'

\section{Discussion \\ Information sharing}

The findings of this study suggest that the most essential need of FMs during the critical illness of a loved one is accessing information. 
FMs' information needs focused on knowing about the patient's care and prognosis and the technical milieu of the ICU. Accessing this information relays anxieties and uncertainties that FMs may have. But, most importantly, FMs felt that information and knowledge about the patient allow them the opportunity to support the patient in the best way possible. The findings clearly illustrate that information is crucial to making sense and gaining control of the situation. Conversely, a lack of information increases negative emotions, leaving FMs in a state of being out of control. Cypress ${ }^{[12]}$ reported that psychosocial support of FMs was fulfilled by the sharing of information, and that providing information to FMs about patient care lessens their anxiety, as they are not familiar with the ICU environment.

In this study, participants verbalised that the information shared should be factual, honest and free from inconsistencies, so as not to create confusion and add to their emotional turmoil. This is consistent with the findings of Mitchell et al. ${ }^{[13]}$ who reported that FMs desired confidence in the nurses caring for the patient and having their questions answered honestly. It is important to provide FMs with a unified message from a cohesive team, especially if there are multiple doctors caring for the patient. ${ }^{[14]}$ Receiving conflicting and contradictory information from different healthcare providers is confusing and worrying. Providing accurate and timely information is respectful; without this information FMs are left in a void that has the potential to impact on them physically and emotionally, leaving them feeling frightened and apprehensive. ${ }^{[15]}$

\section{Reassurance}

The need to be close to the patient was clearly articulated by participants in this study, as they felt they needed to track the care that was being provided. This is supported by Plakas et al. ${ }^{[16]}$ and Gundo et al. ${ }^{[17]}$ who reported that FMs felt a strong need to be close to the patient to see what was happening. These authors also reported on the inter-relationships between vigilant attendance and intense negative emotions. In these studies, FMs reported that they stayed close to the patient because of their anticipatory grief and that whenever they returned home, their anxiety was so great that they wanted to return to the patient's bedside to find relief. Hallgrimsdottir ${ }^{[18]}$ reported that being physically close to the patient comforts the family and helps them to validate the seriousness of the situation and illness. Furthermore, the family's physical separation from the patient is a reminder of the threat to the family system of permanent loss of their loved one.

\section{Striving for consolation}

In our study it emerged that FMs felt that they needed to share their experiences of the sudden unexpected critical illness with other members of the family and with friends, as they thought that their support would alleviate their feelings of suffering. This created a platform for FMs to confront the critical illness as a unit rather than as individuals. Söderström et al. ${ }^{[3]}$ reported that FMs sometimes dissolve into confusion after the sudden admission of a loved one into intensive care. In such situations, FMs gather together, which strengthens the whole family. If coming together is physically impossible, then FMs can use spiritual closeness. These authors suggest that to show feelings of chaos and despair and to share the suffering are a source of consolation, which is vital for family adaptation. However, families who have no tradition of talking openly to each other and discussing their suffering will be negatively affected, which will most likely lead to loneliness, isolation and maladaptation. Striving for consolation constitutes a dynamic and complex adaptation for families, including dynamic relational processes, changing interpersonal relationships, structure and functioning. ${ }^{[3]}$ According to Engström and Söderberg, ${ }^{[19]}$ FMs strive to cope by gathering together and staying close to the critically ill patient.

\section{Garnering of resources}

Results showed that FMs maintained a sense of hope, irrespective of the diagnosis and prognosis of the patient. Despite receiving discouraging information from healthcare professionals, if the patient was warm and could breathe FMs were hopeful. They found that personal cues from the patient signalled such hope. They therefore watched the patient carefully for any kind of reaction, such as the movement of a hand or an eye, interpreting these signs as an awareness of their presence. This supports the findings of the study by Engström and Söderberg, ${ }^{[19]}$ who found that FMs maintained hope that the patient would recover regardless of the prognosis. These authors also indicated that FMs looked for signs of recovery to hold onto, in the hope that the patient would recover. McKiernan and McCarthy ${ }^{[20]}$ mentioned that FMs found reassurance in hope, irrespective of the diagnosis of the patient. Participants believed that it was necessary to cope with the situation.

FMs also verbalised the need to have their physical needs met, which included having a comfortable waiting area. They described the many hours that were spent at the hospital, stressing that comfortable and adequate seating was essential. However, in the context of this study, the comfort needs of the FMs were often neglected as a result of limited resources. Although healthcare professionals acknowledged the importance of meeting these needs, there was not much that could be done about it. According to Jamerson et al., ${ }^{[2]]}$ the waiting area was important to FMs, as they could go there to escape their fears or to cry. Some FMs in this study requested a place to sleep, as they had travelled long distances. This, however, was not always possible, as the ICUs had no family rooms where they could spend the night. In the case of paediatric patients, there were occasions when one of the parents had been allowed to sleep on a chair next to the child.

\section{Cultural and religious co-operation}

From the context of this study, it was evident that healthcare professionals were sensitive to the culture of patients and their FMs. Cultural knowledge is a prerequisite for cultural sensitivity, because awareness of different traditions is essential in caring for people in a multicultural society, such as SA, where inequalities in healthcare systems can lead to stereotyping, prejudice and discrimination. However, to avoid such behaviours, healthcare professionals must become knowledgeable about the world views of different cultures to gain an understanding of them. ${ }^{[22]}$

From data presented in this study, it also became evident that cultural and religious practices provided a source of comfort and feelings of being in control on behalf of FMs. According to Miller ${ }^{[23]}$ spiritual malaise is not conducive to healing, and religious and cultural faith and hope empower an individual to have a perceived sense of control. FMs from diverse cultural groups carried out their traditional rituals in the belief that these practices would assist in ensuring the best outcome for the patient. Similarly, Lundberg and Kerdonfag ${ }^{[24]}$ reiterated that the religious beliefs, practices and cultural rituals of FMs and patients are used as a means of coping with the crisis, promoting healing and recovery or facilitating a peaceful death. In our study, healthcare professionals were faced with numerous religious and cultural rituals. While it is impossible to discuss these in detail, in the context of intensive care, ICNs must take cognisance of varying cultural characteristics such as language, values, behavioural norms, diet, and attitudes toward disease prevention, death and dying, and management of illness. The family 
may view critical illness from a religious or spiritual perspective, and ICNs must try to ensure that the highly technological, illness-focused healthcare system does not clash with cultural and religious beliefs of the patient and FMs. ${ }^{[25]}$

\section{Study limitations}

The study was performed in one province of SA. It could have benefited if more provinces had been included. Moreover, the cultural dynamics in KwaZulu-Natal could differ from those in other provinces.

\section{Conclusion}

We identified five common themes describing the needs of FMs of ICU patients. From these we developed methods that may assist healthcare professionals in providing meaningful support to families.

\section{References}

1. Cypress BS. Understanding the uncertainty among critically ill patients in the intensive care unit using Mishel's theory of uncertainty. Dimens Crit Care Nurs 2016;35(1):42-49. http://dx.doi.org/10.1097/ DCC. 0000000000000152

2. Pryzby B. Effects of nurse caring behaviors on family stress responses in critical care. Intensive Crit Care Nurs 2005;21(1):16-23. http://dx.doi.org/10.1097/10.1016/j.iccn.2004.06.008

3. Söderström I, Saveman B, Hagberg E. Family adaptation in relation to a family member's stay in ICU. Intensive Crit Care Nurs 2009;25(5):250-257. http://dx.doi.org/10.1097/10.1016/j.iccn.2009.06.006

4. Johansson I, Fridlund B, Hildingh C. What is supportive when an adult next of kin is in intensive care ? Nurs Crit Care 2005;10(6):289-98. http://dx.doi.org/10.1097/10.1111/j.1362-1017.2005.00136

5. Bailey J, Sabbagh M, Loiselle C, Boileau J, McVey L. Supporting families in the ICU: A descriptive correlational study of informational support, anxiety and satisfaction with care. Intensive Crit Care Nurs 2010;26(2):114-122. http://dx.doi.org/10.1097/10.1016/j/2009.12.006

6. Söderstrom I, Saveman B, Benzein E. Interactions between family members and staff in intensive care units - an observation and interview study. Int J Nurs Studies 2006;43(6):707-716. http://dx.do org/10.1097/10.1016/j.ijnurstu.2005.10.005

7. Nelms T, Eggenberger $\mathrm{S}$. The essence of the family critical experience and nurse-family meetings. J Fam Nurs 2010;16(4):462-486. http://dx.doi.org/10.1097/10.1177/1074840710386608
8. Hoy S, Severinsson E. Traditional and cultural conflicts for intensive care nurses. J Adv Nurs 2010;66(4):858-867. http://dx.doi.org/10.1097/10.1111/j.1365-2648.2009.05247

9. Strauss A, Corbin I. Basics of Qualitative Research. Grounded Theory Procedures and Techniques. Newbury Park, Calif: Sage Publications, 1990

10. Streubert HJ, Carpenter DR. Qualitative Research in Nursing: Advancing the Humanistic Imperative. Philadelphia, Pa: Lippincott Williams \& Wilkins, 2011.

11. Guba E, Lincoln Y. Naturalistic Inquiry. Newbury Park, Calif: Sage,1995.

12. Cypress BS. The lived ICU experiences of nurses, patients and family members: Phenomenological study with Merleau-Pontian perspective. Intensive Crit Care Nurs 2011;27(5):273-280. http:// dx.doi.org/10.1097/10.1016/j.iccn.2011.08.001

13. Mitchell M, Courtney M, Coyer F. Understanding uncertainity and minimising families' anxiety at the time of transfer from intensive care. Nurs Health Sci 2003;5(3):207-217. http://dx.doi org/10.1097/10.1046/j.1442-2018.2003.00155.x

14. Nelson J, Kinjo K, Meier D, Ahmad K, Morrison R. When critical illness becomes a chronic Informational needs of patients and families. J Crit Care 2005;20(1):79-89. http://dx.doi org/10.1097/10.1016/j.jcrc.2004.11.003

15. Higgins P, Moore S. Levels of theoretical thinking in nursing. Nurs Outlook 2000;48(4):179-183. DOI:10.1067/mno.2000.105248

16. Plakas S, Cant B, Taket A. The experiences of families of critically ill patients in Greece: A socia constructionist grounded theory study. Intensive Crit Care Nurs 2009;25(1):10-20. http://dx.doi. org/10.1097/10.1016/j.iccn.2008.04.003

17. Gundo R, Bodole F, Lengu E, Maluwa A. Comparison of nurses' and families' perception of family needs in critical care unit at referral hospitals in Malawi. Open J Nurs 2014;4(4):312-320. http:// dx.doi.org/10.1097/10.4236/ojn.2014.44036

18. Hallgrimsdottir EM. Accident and emergency nurses' perceptions and experiences of caring for families. J Clin Nurs 2000;9(4):611-619. http://dx.doi.org/10.1097/10.1046/j.1365-2702.2000.00406

19. EngstrÖm A, SÖderberg $S$. The experiences of partners of critically ill persons in an intensive care unit. Intensive Crit Care Nurs 2004;20(5):299-308. http://dx.doi.org/10.1097/10.1016/j.iccn.2004.009

20. McKiernan M, McCarthy G. Family members lived experience in the intensive care unit Phemenological study. Intensive Crit Care Nurs 2010;26(5):254-261. http://dx.doi. org/10.1097/10.1016/j.iccn.2010.06.004

21. Jamerson P, Scheibmeir M, Bott M, Crighton F, Hinton R, Cobb A. The experiences of families with a relative in the intensive care unit. Heart Lung 1996;25(6):467-473. http://dx.doi org/10.1097/10.1016/S0147-9563(96)80049-5

22. Tjale A, de Villiers L. Cultural Issues in Health and Health Care: A Resource Book for Southern Africa. Cape Town: Juta, 2004

23. Miller JF. Coping with Chronic Illness. Overcoming Powerlessness. Philadelphia, Fla: Dans Company, 1983.

24. Lundberg P, Kerdonfag P. Spiritual care provided by Thai nurses in intensive care units. J Clin Nurs 2010;19(7-8):1121-1128. http://dx.doi.org/10.1097/10.1111/j.1365-2702.2009.03072.x

25. Norton C. Caring for the patient's family. In: Fontaine D, Hudak C, Gallo B, eds. Critical Care Nursing: A Holistic Approach. Philadelphia: JB Lippincott, 2005. 